\title{
Thermodynamics of quark matter with saturated confinement interactions *
}

\author{
Ch. Barter \\ Lyman Laboratory of Physics, Harvard University, \\ Cambridge, MA 02138, USA. \\ D. Blaschke ${ }^{\dagger}$ \\ Theory Division, CERN, CH-1211 Geneva 23, Switzerland. \\ H. Voss \\ Fachbereich Physik, Universität Rostock, \\ O-2500 Rostock, FRG.
}

\begin{abstract}
A consistent set of thermodynamical relations for a strongly coupled many-quark system at finite temperature and vanishing baryon number density is derived within the Hartree approximation. Confining interactions are assumed to be saturated within colour singlet quarkantiquark pairs. For a system of massless quarks, simple analytical expressions for the pressure and the energy density are obtained and compared with those of a standard thermodynamical bag model of non-interacting quarks and gluons. We observe that the two equations of state have similar thermodynamical behaviour. A qualitative comparison with results from Lattice Gauge simulations (LGs) shows that the saturation of energy density above the chiral transition can be interpreted as being due to dynamically confined quark matter.
\end{abstract}

CERN-TH.6499/92

July 1992

${ }^{*}$ Supported in part by Deutsche Forschungsgemeinschaft, Grant No. Ro 905/1-1

${ }^{\dagger}$ On leave of absence from Universität Rostock, O-2500 Rostock, FRG. 
Within the description of strongly interacting many-particle systems of quarks and gluons, predictions for the occurrence of phase transitions are of special interest [1]. Up to now, the only way to extract the non-perturbative properties of strongly interacting matter from the first principles of the QCD Lagrangian is to resort to Lattice Gauge simulations (LGs) [2]. However, phenomenological approaches are necessary to interpret the results of LGs and to offer practical tools for prediction and description of phenomena occurring during the quark-hadron phase transitions in the early universe [3] and in experiments with ultrarelativistic heavy-ion collisions [1].

The most popular phenomenological approach is the bag model. Despite the advantages of the bag model's wide applicability and analytic tractability, one is confronted with its inability to describe details of a phase transition. Furthermore, the assumption of an ideal gas of massless quarks and gluons emerging above the chiral transition temperature contradicts the recent observation of correlations ("hadronic wave functions") [4, 5] in LGs. It has been conjectured that quarks above $T_{c}$ might be dynamically confined [6]. For a discussion of these issues, see $[7,8]$.

Effective Lagrangian approaches for low-energy QCD [9] have been developed in order to investigate the thermodynamics of the deconfinement phase transition, including the nonperturbative effects of quark and gluon condensates at finite temperature and density [10]. Within these approaches, the conjecture was formulated that the melting of quark condensates (chiral phase transition) and that of the gluon condensates may not occur simultaneously, thus allowing for a third intermediate phase between the hadronic world and quark-gluon plasma, which is characterized by the dynamical confinement of quarks.

However, the LGs for thermodynamics [2] show a saturation of $\epsilon / T^{4}$ at a constant value and therefore seem to be compatible with the ideal gas assumption of the bag model. In what follows, we show that this behaviour is also found for quark matter with dynamically saturated confinement interactions (string-flip quark matter $=\mathrm{SFQM}$ ).

The description of interacting quark matter within the string-flip model [12] relies on the assumption that within a Mott-type transition the bound states of constituent quarks (hadrons) are 
dissolved at high densities/temperatures by entering a continuum of (colour singlet) scattering states. The confining potential, however, remains unchanged at that transition. The most common choice of such a potential is the Cornell potential:

$$
V(r)=-\frac{\alpha}{r}+\sigma r+V_{0}
$$

By solving the Bethe-Salpeter equation in the instantaneous ladder approximation, with a kernel corresponding to (1), both the heavy and light mesons can be well described [13]. Moreover, it was shown that the introduction of a constant in the kernel of the confinement part for the BetheSalpeter equation does not strongly affect the behaviour of massive mesons, but is essential to generate the low masses of light mesons, see e.g. [13, 14]. Following the arguments of Gromes [15], the constant $V_{0}$ in (1) is related to the string tension $\sigma$ via

$$
V_{0}=-2 \sqrt{\sigma} \exp [-(\gamma-1 / 2)] \approx-2 \sqrt{\sigma} \quad, \quad \gamma=0.577215
$$

Therefore only the strong coupling constant $\alpha=\frac{4}{3} \alpha_{s}$ and the string tension $\sigma$ must be fitted to the hadron spectrum.

However, a direct application of confinement potentials, such as the Cornell type, in deriving the thermodynamical properties of the QCD plasma, leads to several difficulties in evaluating the partition function:

$$
Z=\underset{\{\text { physical states }\}}{\operatorname{Tr}}\left[e^{-\beta\left(H_{0}+V-\mu N\right)}\right] .
$$

All unphysical states (colour van der Waals forces etc.) must be removed from the theory, and suitable renormalization procedures (similar to the case of screening in Coulomb plasmas) need to be applied to avoid divergences. One possible scheme is saturation of the confinement interaction (1) which has been realized within the string-flip model of quark matter [17] and hadron-hadron scattering [18]. However, the mathematical treatment of colour saturation in a many-particle system is a very difficult task and Monte Carlo techniques have been applied [19] to solve it at near-zero temperature. The aim of the present paper is threefold:

1. To treat the colour saturation problem within the nearest-neighbour approach [12] which provides us with analytic results for a density-dependent potential energy of quark matter. 
2. To derive a consistent set of thermodynamical relations for quark matter at $\mu=0$ (vanishing baryon number density).

3. To apply the result to massless quarks (above the chiral transition) and perform a comparison with the well-known bag model and (qualitatively) with LGs.

This approach is similar to strong coupling phenomena in solid-state physics, such as electronhole recombination, where an effective potential is constructed from a naked interaction by weighting each interquark distance $r$ (string length) with the probability of finding the nearest neighbour of corresponding colour there, i.e.

$$
V^{e f f}(r, n)=V(r) c(r, n)
$$

where

$$
c(r, n)=\exp \left[-\frac{4 \pi r^{3} n}{3 N_{c}}\right],
$$

is the unnormalized nearest-neighbour distribution function in three dimensions for uncorrelated quarks with the number density $n=n_{q}=n_{\bar{q}}$, and $N_{c}$ is the number of colours, (see e.g. [12]).

The potential energy density corresponding to the effective potential (1) is given by

$$
u(n)=\frac{n^{2}}{N_{c}} \int_{\Omega} V^{e f f}(r, n) d^{3} r .
$$

For interactions $V(r)$ of the general polynomial form $V(r)=\sum_{k} a_{k} r^{b_{k}}, b_{k} \in R$ the potential energy density can be evaluated analytically. One obtains for the Cornell potential (1):

$$
u(n)=V_{0} n+\sigma \Gamma\left(\frac{4}{3}\right)\left(\frac{3 N_{c}}{4 \pi}\right)^{1 / 3} n^{2 / 3}-\alpha \Gamma\left(\frac{2}{3}\right)\left(\frac{3 N_{c}}{4 \pi}\right)^{-1 / 3} n^{4 / 3},
$$

with $\Gamma\left(\frac{2}{3}\right)=1.35412, \Gamma\left(\frac{4}{3}\right)=0.89298$. The equation of state for a system of particles obeying a density-dependent interaction energy density has recently been investigated by Zimanyi et al. [20] for the case of nuclear matter in the Walecka model approach, and has since been utilized by several authors (see $[21,22,23])$. Within these approaches, the grand canonical partition function is constructed from $u(n)$ on the Hartree-Fock level, so that all relevant thermodynamical relations 
are systematically obtained as partial derivatives, which thereby gurantees their self-consistency. The pressure, for example, is given by [21]

$$
\begin{aligned}
P(T) & =T \Omega^{-1} \ln Z \\
& =2 \frac{N_{f} N_{c}}{3 \pi^{2}} \int_{0}^{\infty} d p\left[\frac{p^{4}}{E(p)}\left\{1+\exp \left[\left(E(p)+\Delta^{H}\right) / T\right]\right\}^{-1}\right]-u(n)+2 n \Delta^{H}(n),
\end{aligned}
$$

with

$$
E(p)=\sqrt{p^{2}+m^{2}}
$$

as the free one-particle energy, where $N_{f}$ are the flavour degrees of freedom, and the chemical potentials are set to zero (resulting in zero baryon number). The Hartree shift is given by

$$
\Delta^{H}(n)=\left.\frac{\partial u\left(n^{\prime}\right)}{\partial n^{\prime}}\right|_{n^{\prime}=n}
$$

For the energy density we find the following expression

$$
\begin{aligned}
\epsilon(T)= & -T^{2} \frac{\partial}{\partial T}\left(\frac{P}{T}\right) \\
= & 2 \frac{N_{f} N_{c}}{\pi^{2}} \int_{0}^{\infty} d p\left[p^{2} E(p)\left\{1+\exp \left[\left(E(p)+\Delta^{H}\right) / T\right\}^{-1}\right]\right. \\
& +u(n)+T \Delta^{H}(n) \frac{\partial n}{\partial T} .
\end{aligned}
$$

To our knowledge, this is the first time that a thermodynamically-consistent description of quark matter at the Hartree-Fock level has been presented. Earlier attempts $[24,25]$ to implement confinement interactions within the Hartree-Fock scheme do not satisfy these requirements.

We now examine thermodynamics in the region

$$
\left|\frac{\Delta^{H}}{T}\right| \ll 1,
$$

so that the partition function (3) can justifiably be Taylor-expanded with respect to this small quantity. Such an expansion should also lead to a thermodynamically consistent set of relations. Utilizing our approximation, we obtain, to first order in $\Delta^{H} / T$ :

$$
P(T)=P^{i d}(T)-u\left(n^{i d}\right)
$$


By considering the pressure (8) as a thermodynamical potential, the other state variables can be easily derived; in particular, for the energy density, $\epsilon(T)$

$$
\epsilon(T)=\epsilon^{i d}(T)+u\left(n^{i d}\right)-\Delta^{H}\left(n^{i d}\right)\left(3 n^{i d}+\frac{2 N_{f} N_{c}}{\pi^{2}} m^{2} \int_{0}^{\infty} d p\{1+\exp [E(p) / T]\}^{-1}\right)
$$

and the entropy density $s(T)$

$$
T s(T)=P^{i d}(T)+\epsilon^{i d}(T)-\Delta^{H}\left(n^{i d}\right)\left(3 n^{i d}+\frac{2 N_{f} N_{c}}{\pi^{2}} m^{2} \int_{0}^{\infty} d p\{1+\exp [E(p) / T]\}^{-1}\right) .
$$

The desired thermodynamical consistency condition is demonstrated by substituting the expanded quantities into the Euler relation

$$
\epsilon(T)=T s(T)-P(T)
$$

The pressure which is defined by eq. (13) was previously used for investigations of quark-matter systems within the string-flip model, see e.g. [26], where, in particular, a comparison was performed with parametrizations of this quantity within the bag model approach. However, the energy density (14) is given here for the first time.

In the following, we perform the calculations with massless quarks, consistent with the assumption of chiral symmetry in the SFQM, where no pions can be formed. In this case, we have

$$
n_{q}^{i d}(T)=a T^{3} \quad, \quad a=\frac{3 \zeta(3)}{2 \pi^{2}} N_{f} N_{c}
$$

and the potential energy density (6) can be given as a function of the temperature. For the Cornell potential (1), we obtain

$$
u\left(n^{i d}\right)=u(T)=\tilde{\sigma} T^{2}+\tilde{V}_{0} T^{3}-\tilde{\alpha} T^{4},
$$

where we have introduced the following abbreviations

$$
\begin{aligned}
\tilde{\sigma} & =\sigma \Gamma\left(\frac{4}{3}\right) a\left(\frac{3 N_{c}}{4 \pi a}\right)^{1 / 3} \\
\tilde{V}_{0} & =V_{0} a \\
\tilde{\alpha} & =\alpha \Gamma\left(\frac{2}{3}\right) a\left(\frac{3 N_{c}}{4 \pi a}\right)^{-1 / 3} .
\end{aligned}
$$

Inserting

$$
P^{i d}(T)=\frac{7 \pi^{2}}{180} N_{f} N_{c} T^{4}
$$


and (18) into (13) and (14), we obtain for the pressure and energy density of the interacting quark matter phase

$$
\begin{aligned}
P(T) & =\frac{\pi^{2}}{90}\left(\frac{7}{2} N_{f} N_{c}+\frac{90}{\pi^{2}} \tilde{\alpha}\right) T^{4}-\tilde{\sigma} T^{2}-\tilde{V}_{0} T^{3} \\
\epsilon(T) & =3 P(T)+\delta(T)
\end{aligned}
$$

where the interaction measure $\delta(T)$ is given by

$$
\delta(T)=T^{5} \frac{d}{d T}\left(\frac{P}{T^{4}}\right)=2 \tilde{\sigma} T^{2}+\tilde{V}_{0} T^{3} .
$$

In Fig. 1 we display the individual contributions of the Cornell potential (1) to the pressure (21) for the parameter set quoted below equation (26). We note that when the string tension $\sigma$ is turned off, no phase transition can occur.

Representing the hadronic phase by a gas of non-interacting massless pions,

$$
P_{h}(T) \equiv P_{\pi}(T)=\frac{\pi^{2}}{90} g_{\pi} T^{4}
$$

and requiring the Gibbs criterion of pressure equilibrium, $P_{H} \equiv P_{h}\left(T_{c}\right)=P_{Q} \equiv P_{q}\left(T_{c}\right)$, the critical temperature for the transition from hadronic matter to colour-saturated quark matter is obtained from the solution of a quadratic equation in $T$,

$$
T_{c}=c \sqrt{\sigma}
$$

with the introduced abbreviations

$$
\begin{aligned}
c & =\frac{a}{c^{\prime}}\left(\sqrt{1+\frac{c^{\prime}}{a} \Gamma\left(\frac{4}{3}\right)\left(\frac{3 N_{c}}{4 \pi a}\right)^{1 / 3}}-1\right), \\
c^{\prime} & =\frac{\pi^{2}}{90}\left(\frac{7}{2} N_{f} N_{c}-g_{\pi}\right)+\tilde{\alpha} .
\end{aligned}
$$

Here, we have made use of relation (2) between the constant $V_{0}$ and the string tension $\sigma$. The parameters of the Cornell potential (1) $\alpha_{s}=0.583, \sigma=0.27 \mathrm{GeV}^{2}$ are adjusted to fit the hadron mass spectra at $T=0$ with $m_{q}=0.32 \mathrm{GeV}$ as described in [16]. In the case of massless quarks considered above, a critical temperature $(25) T_{c}=146 \mathrm{MeV}$ is obtained for the chiral phase transition. 1

\footnotetext{
${ }^{1}$ Increasing the number of massless flavors to $N_{f}=4$ lowers the critical temperature to $T_{c}=118 \mathrm{MeV}$ in accordance with LGs.
} 
A calculation with massive quarks $m_{q}=0.32 \mathrm{GeV}$ gives $T_{c}=178 \mathrm{MeV}$ (see also [26]). However, the massive quark matter phase is thermodynamically disfavoured as can be seen in Fig. 1.

In contrast to [24], our systematic derivation of the potential energy density $u(n)$ within the colour saturation scheme gives a critical temperature $T_{c}$ well in accordance with recent lattice calculations for dynamical fermions $N_{f}=2[2,27,28]$.

Furthermore, for the advocated parameter set, the Hartree shift becomes zero at $T_{0} \approx 140 \mathrm{MeV}$, so that the condition (12) for the applicability of the above linear-shift thermodynamics is fulfilled just in the region of the phase transition where the approach is applied.

We now wish to compare the thermodynamics of colour-saturated quark matter with that of non-interacting quarks and gluons in a thermodynamical bag model. In the latter, the relations corresponding to (21), (23) and (25) are

$$
\begin{aligned}
P_{b a g}(T) & =\frac{\pi^{2}}{90}\left(\frac{7}{2} N_{f} N_{c}+2 N_{g}\right) T^{4}-B \\
\delta_{b a g}(T) & =4 B \\
T_{c}^{b a g} & =B^{1 / 4}\left[\frac{\pi^{2}}{90}\left(\frac{7}{2} N_{f} N_{c}+2 N_{g}-g_{h}\right)\right]^{-1 / 4} .
\end{aligned}
$$

To compare these results with those from LGs, we introduce the scaled temperature $T / T_{c}$. The results for energy density and pressure are displayed in Fig. 2.a. We observe a strong similarity between the bag model for a gas of non-interacting massless quarks and gluons and the present string-flip model for colour-singlet quark matter with saturated confinement interactions. This behaviour is interesting since the roughly constant value of $\epsilon / T^{4}$, obtained in LGs just above $T_{c}$, is traditionally interpreted as a measure of the number of elementary "degrees of freedom" (Stefan-Boltzmann limit). These are quite different in the two models. ated Our accounting for the colour saturated interactions within the quasi-particle Hartree-Fock approach mimics the "degrees of freedom" of free gluons which are absent in SFQM. This is enhancement explicitly arises in the pressure formula (13).

The enhancement of degrees of freedom due to interactions is typical of any Beth-Uhlenbeck approach. However, as has been shown for nuclear matter in [29], the classical Beth-Uhlenbeck formula breaks down at the Mott density, above which a quasi-particle picture for the elementary 
degrees of freedom takes over. Such a generalized Beth-Uhlenbeck approach remains to be worked out for quark matter.

In Fig. 2.a we have also shown, as a qualitative comparison, the results of LGs for $S U(3)_{c}$ with $N_{f}=2$ fermions [27] for pressure and energy density (scaled by an overall factor, see [30]). These show that dynamically confined quark matter explains the data as well as the bag model.

It is interesting to note that both in the SFQM and the bag model the interaction measure $\delta=\epsilon-3 P$ is non-perturbative scale-free, that is, the parameters $B$ and $\sigma$ drop out respectively when using the scaled temperature. Thus, $\delta$ lends itself particularly well for a qualitative comparison of the two models, as depicted in Fig. 2.b. From the results for the interaction measure $\delta$, it can be seen that the transition region $T \simeq T_{c}$ is not adequately described in both models. This will be discussed below. We also observe that massive SFQM given in Fig. 2.b clearly fails to model the LGs data above $T_{c}$.

Note that the limit for SFQM will be reached at a critical temperature $T^{*}$ above which perturbative QCD is a thermodynamically favourable description of quark-gluonic matter. This phase arises due to the temperature dependence of the strong coupling constant $\alpha(T)$. In the phenomenological bag-model description of this phase given by Eq. (27), the bag-constant is calculated to be $B^{1 / 4} \sim 240 \mathrm{MeV}$ from QCD sum rules [31]. We emphasize that a three-phase description with an intermediate SFQM stage could serve as a realistic phenomenology for a description of the hadronic to quark-gluonic matter transition.

Since the thermodynamical string-flip model departs from a microscopic description of hadronhadron scattering on the quark level, it can be used, for example, to estimate reaction rates of hadrons traversing the quark matter phase. It thus provides theoretical tools to investigate signals for the deconfinement transition, such as heavy quarkonia suppression, (see [32] and references cited therein).

However, the details of the Mott-type transition from hadronic matter to colour-saturated quark matter remain to be worked out:

1. The Hartree-Fock level of description for the two-quark correlation function is expected to break down at $T \simeq T_{c}$, where a proper formulation of bound-state formation has to be given, via e.g. a thermodynamical $T$-matrix approach. This work is in progress. 
2. We expect that the three-phase description of the deconfinement transition, for the case of vanishing baryochemical potential $\mu=0$, is valid in the entire $T-\mu$ phase diagram. The generalization of the string-flip model to the case $\mu>0$ should be possible along the lines of [12]. This holds especially in the $T=0$ case [33] for which the string-flip model was originally developed [17, 12]. The calculation for $T>0, \mu>0$ would be of interest, for example, in estimating the density contrast emerging in the the Big Bang hadronization transition independently of the bag model calculations [3]. This is worth doing since differently chosen bag models lead to arbitrariness in the results, which could be circumvented within the suggested potential model approach.

3. A proper inclusion of the density dependence of the quark and gluon condensates, as, for instance, within an effective Lagrangian approach [10] which describes the behaviour $\sigma(T) \rightarrow$ 0 leading to deconfinement (asymptotic freedom), is deferred to future work.

In conclusion, we have presented the thermodynamics of quark matter with colour- saturated confinement interactions in an effective approach consistent with a Hartree-Fock level of description. Our main result is that dynamical confinement of quarks above the chiral transition temperature effectively enhances the "degrees of freedom" and is consistent with the thermodynamics obtained from Monte Carlo simulations of Lattice Gauge Theory.

\section{Acknowledgements}

We acknowledge the hospitality of the Theory Division at CERN, where this work has been completed. One of us (D.B.) is indebted to G. Brown and to H. Satz for many stimulating discussions.

\section{References}

[1] H. Satz, Proc. Joint Lepton-Photon Symposium and Europhysics Conference on High Energy Physics, Geneva, 1991 (World Scientific, Singapore, 1992), p. 273.

[2] F. Karsch, Z. Phys. C38 (1988) 147. 
[3] K. Kajantie and H. Kurki-Suonio, Phys. Rev. D34 (1986) 1719.

J.H. Applegate, C.J. Hogan and J. Scherrer, Phys. Rev. D35 (1987) 1151.

C. Alcock, G.M. Fuller and G.J. Mathews, Astrophys. J. 320 (1987) 439.

[4] C. Bernard, M.C. Ogilvie, C. DeTar, S. Gottlieb, A. Krasnitz, R.K. Sugar and D. Toussaint, Univ. Arizona Report AZPH/91-60 (1991).

[5] K.D. Born, S. Gupta, A. Irbäck, F. Karsch, E. Laermann, B. Peterson and H. Satz, Phys. Rev. Lett. 67 (1991) 302.

[6] C. DeTar, Phys. Rev. D37 (1988) 2328.

[7] G.E. Brown, A.D. Jackson, H.A. Bethe and P.M. Pizzochero, Stony Brook preprint, SUNY NTG-92-06 (1992).

[8] V. Koch, E.V. Shuryak, G.E. Brown and A.D. Jackson, Stony Brook preprint, SUNY NTG$92-4(1992)$.

[9] B.A. Campbell, J. Ellis and K.A. Olive, Nucl. Phys. B345 (1990) 57.

[10] J. Ellis, J.I. Kapusta and K.A. Olive, Phys. Lett. B273 (1991) 123. G. Ripka and M. Jaminon, Saclay preprint SPhT/92-018 (1992).

[11] M.I. Gorenstein and O.A. Mogilevsky, Z. Phys. C38 (1988) 161.

[12] G. Röpke, D. Blaschke and H. Schulz, Phys. Rev. D34 (1986) 3499.

[13] C.-Y. Habe, Phys. Rev. D44 (1991) 2928.

[14] F. Gross and J. Milana, Phys. Rev. D43 (1991) 2401.

[15] D. Gromes, Z. Phys. C11 (1981) 147, C14 (1982) 94 Erratum.

[16] W. Lucha, F. Schöberl and D. Gromes, Phys. Rep. 200 (1991) 127.

[17] C.J. Horowitz, E. Moniz and J. Negele, Phys. Rev. D31 (1985) 1689.

[18] F. Lenz et al., Ann. Phys.(NY) 170 (1986) 65. 
[19] C.J. Horowitz and J. Piekarewicz, Univ. Indiana preprint IU/NTC 91-04 (1991).

[20] J. Zimanyi, B. Lukacs, P. Levai, J. Bondorf and N.L. Balacs, Nucl. Phys. A484 (1988) 647.

[21] D.H. Rischke, B.L. Friman, H. Stöcker and W. Greiner, J. Phys. G14 (1988) 191.

[22] H.W. Barz, B.L. Friman, J. Knoll and H. Schulz, Phys. Rev. D40 (1989) 157.

[23] K.A. Bugaev, M.I. Gorenstein, B. Kämpfer and V.I. Zhdanov, Phys. Rev. D40 (1989) 2903.

[24] K.A. Olive, Nucl. Phys. B190 (1981) 483 and B198 (1982) 461.

[25] D. Blaschke, G. Röpke, F. Reinholz and D. Kremp, Phys. Lett. B151 (1985) 439.

[26] H. Voss, D. Blaschke, G. Röpke and B. Kämpfer, J. Phys. G15 (1989) 561.

[27] S. Gottlieb et al., Phys. Rev. D35 (1987) 3972.

[28] F. Karsch, Plenary talk given at the DESY Theory Workshop, Hamburg, Sept. 30 - Oct. 2, 1991.

[29] M. Schmidt, G. Röpke and H. Schulz, Ann. Phys. 202 (1990) 57.

[30] F. Karsch and I.O. Stamatescu, Phys. Lett. B 227 (1989) 153.

[31] M.A. Shifman, A.I. Vainshtein and V.I. Zakharov, Nucl. Phys. B147 (1979) 385 and B147 (1979) 448.

[32] D. Blaschke, Nucl. Phys. A525 (1991) 269c.

[33] H. Schulz and G. Röpke, Z. Phys. C35 (1986) 379 


\section{Figure captions}

\section{Figure 1:}

The pressure $P$ normalized by $T^{4}$ as a function of temperature for the massless string-flip model of quark matter (full line). The contributions of the ideal massless quarks and antiquarks (dotteddashed line) and of the one-gluon exchange (dashed line) are also shown. The dotted line represents the massive case of the string-flip model. The arrow indicates the flat Stefan-Boltzmann limit.

Figure 2:

(a) The pressure $P$ (lower curves) and the energy density $\epsilon$ (upper curves) normalized by $T^{4}$ as a function of $T / T_{c}$. The full line represents colour singlet quark matter within the string-flip model and the dashed line massless non-interacting quarks and gluons within the standard bag model. At $T=T_{c}$ there is pressure equilibrium with a phase of massless ideal pions. Lattice QCD results (LGs) for $N_{f}=2[27]$ are shown for a qualitative comparison. They are scaled by an overall factor, see Ref. [2]. The arrow indicates the Stefan-Boltzmann limit $\epsilon_{S B} \simeq 12.2 T^{4}$. For the choice of the parameters, see text.

(b) The interaction measure $\delta$ normalized by $T^{4}$ as a function of $T / T_{c}$ for the string-flip model with massless quarks (full line) and the bag model (dashed line). Both models are very similar but fail to describe the details at $T \sim T_{c}$. The results from LGs (dots) correspond to those in Fig. 2.a. The dotted line shows the string-flip model with massive quarks $m_{q}=0.32 \mathrm{GeV}$. 


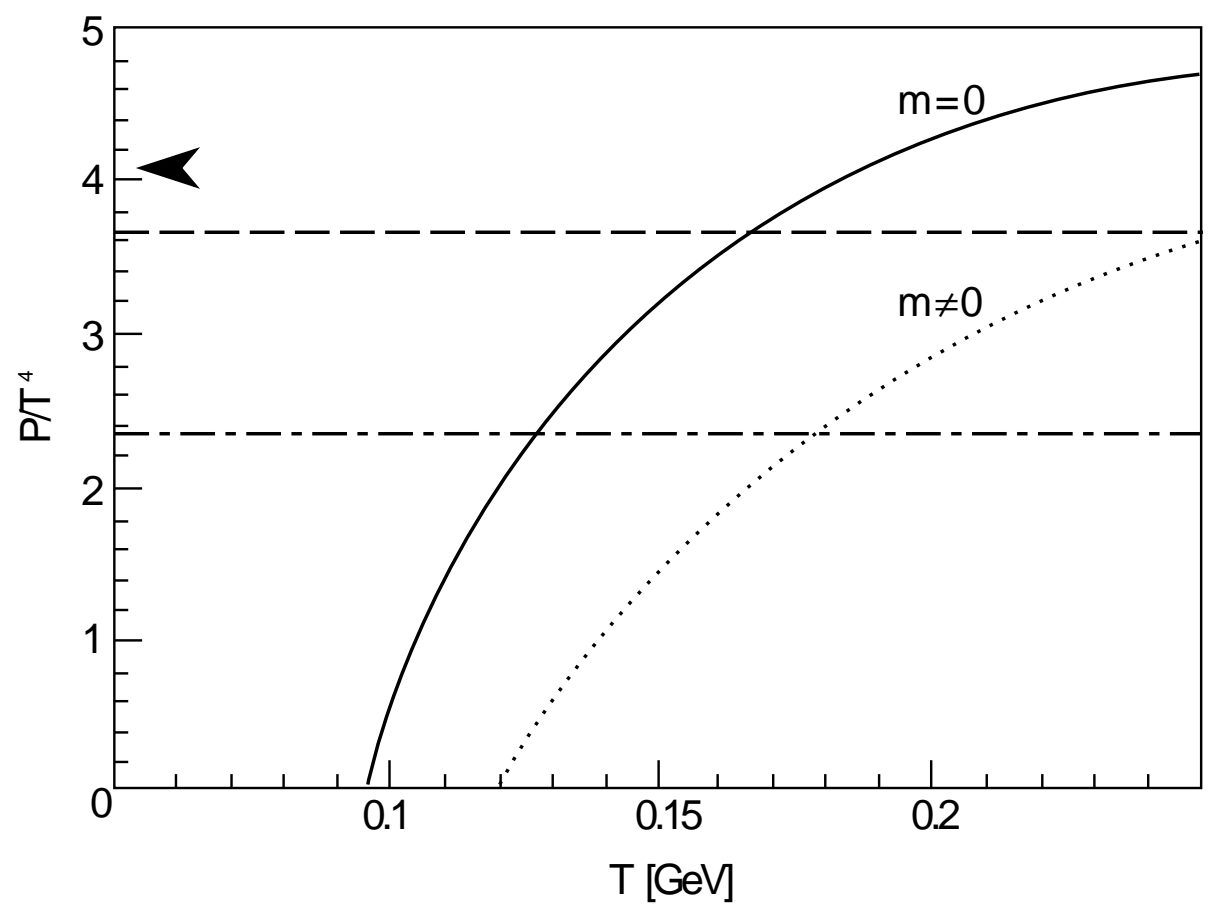

Fig. 1 


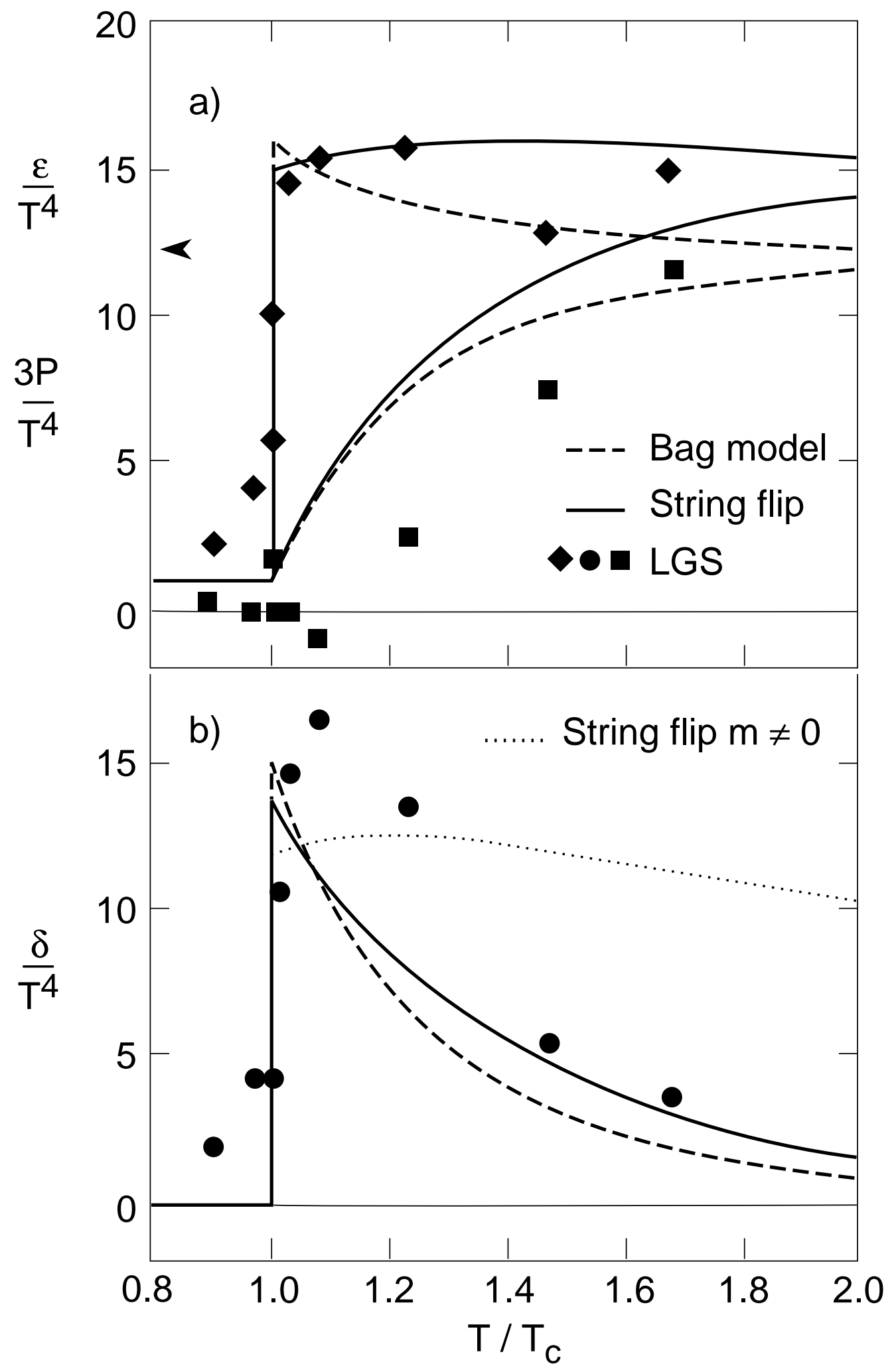

Fig. 2 